\section{ORIGINAL RESEARCH}

\author{
K.D. Bodily \\ H.J. Cloft \\ G. Lanzino \\ D.J. Fiorella \\ P.M. White \\ D.F. Kallmes
}

\title{
Stent-Assisted Coiling in Acutely Ruptured Intracranial Aneurysms: A Qualitative, Systematic Review of the Literature
}

\begin{abstract}
BACKGROUND AND PURPOSE: The use of stents for treatment of morphologically unfavorable, acutely ruptured aneurysms is avoided by most operators because of concerns about the risk of using dual antiplatelet therapy in the setting of acute SAH. Our aim was to review the literature regarding stent-assisted coil embolization of acutely ruptured intracranial aneurysms to determine the safety and efficacy of this treatment option.
\end{abstract}

MATERIALS AND METHODS: Articles including $\geq 5$ patients with ruptured aneurysms who were treated acutely with stent-assisted coiling or uncovered stent placement alone were identified. Data on clinical presentation, technical success, surgical crossover, intracranial complications, and clinical outcome were evaluated.

RESULTS: A total of 17 articles were identified reporting 339 patients who met inclusion criteria. Among 212 patients with available data, technical success was noted in 198 (93\%) patients. Three hundred twenty-six (96\%) of 339 patients received both heparin during the procedure and dualantiplatelet therapy during or immediately postprocedure. One hundred thirty (63\%) of 207 aneurysms were completely occluded. Six (2\%) of 339 patients required surgical crossover, usually for failure in stent placement or for intraprocedural aneurysm rupture. Clinically significant intracranial hemorrhagic complications occurred in $27(8 \%)$ of 339 patients, including $9(10 \%)$ of 90 patients known to have EVDs who had ventricular drain-related hemorrhages. Clinically significant thromboembolic events occurred in $16(6 \%)$ of 288 patients. Sixty-seven percent of patients had favorable clinical outcomes, $14 \%$ had poor outcomes, and $19 \%$ died.

CONCLUSIONS: Stent-assisted coiling in ruptured aneurysms can be performed with high degrees of technical success, but adverse events appear more common and clinical outcomes are likely worse than those achieved without stent assistance. Thromboembolic complications appear reasonably well-controlled. Reported EVD-related hemorrhagic complications were uncommon, though the total number of EVDs placed was unknown.

ABBREVIATIONS: EVD = external ventricular drain; GOS = Glasgow Outcome Score; $\mathrm{HH}=$ Hunt and Hess; $\mathrm{mRS}=$ modified Rankin Score; $\mathrm{SAH}=$ subarachnoid hemorrhage

$S^{t}$ tent-assisted coiling is a widely applied technique for treatment of wide-neck aneurysms. ${ }^{1-5}$ However, to mitigate the risk of stent-associated thromboembolic complications, dual antiplatelet therapy is used routinely pre-, peri-, and postoperatively. ${ }^{6-8}$ Because of the need for dual antiplatelet therapy and concerns regarding the use of antiplatelet therapy in patients with acute $\mathrm{SAH}$, the use of stent-assisted coiling has largely been reserved for patients with unruptured aneurysms. ${ }^{9-14}$ Use of the stent-assisted technique for ruptured aneurysms is considered by many practitioners to expose patients to elevated risk of bleedingrelated complications if interventions such as EVD placement or ventriculoperitoneal shunt surgery are subsequently required..$^{9-12,15,16}$

\section{Received April 15, 2010; accepted after revision November 18}

From the Departments of Radiology (K.D.B., H.J.C., G.L., D.F.K.) and Neurosurgery (G.L.), Mayo Clinic, Rochester, Minnesota; Department of Neurosurgery (D.J.F.), Stony Brook Medical Center, Stony Brook, New York; and Division of Clinical Neurosciences (P.M.W.), Western General Hospital, Edinburgh, United Kingdom.

Please address correspondence to David F. Kallmes, MD, Department of Radiology, Mayo Clinic, 200 First St SW, Rochester, MN 55905; e-mail: kallmes.david@mayo.edu

Indicates open access to non-subscribers at www.ajnr.org

DOI 10.3174/ajnr.A2478
Notwithstanding the reluctance of many operators to use stent-assisted coiling in cases of ruptured aneurysms, a number of case series of stent use in ruptured aneurysms have now been published. ${ }^{6,9-13,17-26}$ Review of these published case series might inform the neurovascular community regarding periprocedural management options as well as rates of hemorrhagic complications associated with stent-assisted coiling in ruptured aneurysm cases. The purpose of the current study was to summarize existing literature data to inform the community regarding the safety and efficacy of stent-assisted coiling in acutely ruptured aneurysms.

\section{Materials and Methods}

The relevant neuroradiologic and neurosurgical literature was searched via the PubMed and Ovid data bases up to and including February 2010 by using the following search terms: "aneurysm," "aneurysms," or "endovascular" and text words including "intracranial" or "cerebral" or "carotid" or "basilar" and "stent" and "rupture" or "ruptured" or "hemorrhage" or "hemorrhagic." We excluded articles with titles including the words "aorta" or "aortic" or "coronary." Identified articles were then evaluated for reports of aneurysm treatment using uncovered stents, either stent-assisted coiling or stents alone. All articles that included treatment of at least 5 acutely ruptured 


\begin{tabular}{lc}
\hline Table 1: Summary of patients & \\
\hline Patients and Publications & Data \\
\hline Publications & 17 \\
Patients treated with stents acutely (<7days) after & 339 \\
$\quad$ aneurysm rupture & $8(2 \%)$ \\
Patients treated with stents alone & $331(98 \%)$ \\
Patients treated with stents and coils & $326(96 \%)$ \\
\hline
\end{tabular}

aneurysms with uncovered stents were collected. Dissecting aneurysms were excluded, but articles reporting blood blister-like aneurysms were included. Articles including patients treated acutely with stents were excluded if these stents were used exclusively as a bailout technique, because the conditions of these patients are inherently more complicated with patients having a higher probability of worse outcomes. Articles that met inclusion criteria were then evaluated to collect data regarding 3 general categories: technical success, intracranial complications, and clinical outcomes. While multiple articles reported stent-assisted treatment of both elective, nonruptured and acutely ruptured aneurysms in the same report, only those that specified data for the acutely ruptured cases in at least 1 of the above 3 categories were included.

Technical success was measured in various ways by different authors. Data were collected regarding successful placement of stents, as reported by each author; use of stents with coils or stents alone; and aneurysms occlusion rates. Data regarding the need for subsequent surgical intervention including open aneurysm repair or the need for EVD placement were also collected.

Aneurysm occlusion rates were reported in various ways in 11 of 17 articles, representing 207 (61\%) of 339 patients, 6,9,10,12,17,19,21-24 including categories such as "complete occlusion," "neck remnant," "partial occlusion," and "no occlusion." Three of 17 articles s, $^{9,25}$ quantified occlusion as $100 \%,>95 \%,>90 \%,<90 \%$, and so forth, which was converted to the 4-point graded system above.

Clinical outcomes were reported in various ways and were available in 13 of 17 articles representing 293 (86\%) of 339 patients. ${ }^{6,9,10,12,17,19-22,24-27}$ Seven articles used the mRS, and 6 articles used the GOS. "Good outcome" was considered mRS 0-2 or GOS 1-2. "Poor outcome" was considered mRS 3-5 or GOS 3-4. Because clinical outcomes are highly dependent on preprocedural clinical conditions, these data were evaluated in the context of the degree of neurologic compromise at the time of presentation, which was usually reported by using the $\mathrm{HH}$ grading system in 13 of 17 articles representing 305 (90\%) of 339 patients.

All reported intracranial complications within the first 30 days after the procedure were included in this evaluation. By definition, complications had to result in clinical sequelae, either temporary or permanent, or had to result in additional therapy of some kind. When complications were listed but clinical sequelae were not specifically reported or if clinical outcome could have been affected by the complication, this complication was considered clinically significant to avoid underestimation of the clinical impact of complications.

\section{Results}

A total of 56 articles were identified (December 1997 to February 2010) that reported a combined total of 428 patients who were treated acutely with stents or stent-assisted coiling. Of these, 17 (30\%) articles, all published between June 2004 and February 2010, met inclusion criteria for this analysis (Table 1). ${ }^{6,9-13,17-27}$ These 17 articles reported a combined total of 339 patients (range $=5-61$ patients per article, mean $=21$ patients, median $=14$ patients) who were treated acutely with stents or stent-assisted coiling. All 17 reports were retrospective case series. Thirty-nine $(70 \%)$ of 56 articles were excluded because the report contained $<5$ cases $(n=28)$, the patients were not treated in the acute phase $(n=6)$, the report consisted of dissecting aneurysms $(n=10)$, data on outcomes were not provided $(n=1)$, all of the patients were bailout cases $(n=1)$, or covered stents were used $(n=1)$. Fourteen $(82 \%)$ of 17 included reports also had data on patients with unruptured aneurysms; these latter patients were excluded from this current analysis.

Technical approaches varied. Fourteen of 17 articles representing 267 (79\%) of 339 patients reported a single intervention for placement of an uncovered stent and platinum MicroCoils (Micrus, San Jose, California) to treat wide-neck aneurysms. $6,9,10,12,13,17-19,21,23-27$ Three of 17 articles representing $72(21 \%)$ of 339 patients had a subset of procedures that were staged, but only patients who had uncovered stents placed acutely were included in this analysis. ${ }^{11,20,22}$ Eight (2\%) of 339 patients had uncovered stent placement alone without coil placement. ${ }^{6,17,19}$

A number of different stent manufacturers were included in the series. Three hundred four (90\%) of 339 patients received Neuroform I, II, or III microstents (Boston Scientific, Natick, Massachusetts), ${ }^{6,9,10,12,13,17-20,22,23,25-27}$ while 16 (5\%) of 339 received the Enterprise self-expanding stent (Cordis, Miami Lakes, Florida). ${ }^{11}$ Seven (2\%) of 339 received the Solitaire AB stent (ev3, Irvine, California), ${ }^{21}$ and 12 (4\%) of 339 received either Neuroform or Wingspan stent (Boston Scientific), not specified. ${ }^{24}$

All authors reported the use of anticoagulation, but methods varied among studies. None of the patients received antiplatelet therapy or heparin before the procedure. All patients received heparin during the procedure. In 326 (96\%) of 339 cases, dual antiplatelet therapy, usually aspirin and clopidogrel, was administered after the procedure; clopidogril was continued for approximately 3 months postprocedure and aspirin, from 3 months to indefinitely. ${ }^{6,9-13,17-25,27}$ Minor variations in anticoagulation/antiplatelet therapy included the use of heparin without bolus infusion, reversal of heparin at the end of the procedure versus continuation for 24-48 hours postprocedure, the use of only 1 antiplatelet agent, delaying antiplatelet therapy until the morning after the procedure, variations in the duration of the antiplatelet therapy in general, and the use of glycoproteins IIb/IIIa inhibitors in a few select patients.

Details regarding technical success were provided in 212 (63\%) of 339 patients. ${ }^{6,9,10,12,17-19,21-26}$ Among these 212 cases, technical success was noted in 197 (93\%) patients. Angiographic occlusion data were provided in 206 (61\%) of 339 patients. One hundred thirty patients $(63 \%)$ of 206 had complete occlusion, 39 patients (19\%) of 206 had a neck remnant, and 26 patients $(13 \%)$ of 206 had residual patency in the aneurysm. Eleven patients (5\%) of 206 had no occlusion. Six (2\%) of 339 patients had endovascular treatment failure of some kind that required subsequent open surgical intervention (Table 2).

Bleeding complications are shown in Table 3. Intracranial hemorrhage related to EVD placement was reported in a total 


\begin{tabular}{|c|c|c|c|}
\hline Technical Success & Patients & $\begin{array}{c}\% \text { for } \\
\text { Patients } \\
\text { with Data }\end{array}$ & $\begin{array}{c}\text { Proven } \% \text { for } \\
\text { Total } 339 \\
\text { Patients }\end{array}$ \\
\hline $\begin{array}{l}\text { Successful stent deployment } \\
\text { and placement }\end{array}$ & 198 & $93 \%(212)$ & $58 \%$ \\
\hline $\begin{array}{l}\text { Patients requiring subsequent } \\
\text { surgical intervention }\end{array}$ & 6 & $2 \%(301)$ & $2 \%$ \\
\hline $\begin{array}{l}\text { Raymond class I occlusion (or } \\
\text { equivalent) }\end{array}$ & 130 & $63 \%(207)$ & $38 \%$ \\
\hline $\begin{array}{l}\text { Raymond class II occlusion } \\
\text { (or equivalent) }\end{array}$ & 39 & $19 \%(207)$ & $12 \%$ \\
\hline $\begin{array}{l}\text { Raymond class III occlusion } \\
\text { (or equivalent) }\end{array}$ & 26 & $13 \%(207)$ & $8 \%$ \\
\hline No occlusion & 11 & $5 \%(207)$ & $3 \%$ \\
\hline
\end{tabular}

\begin{tabular}{lccc}
\hline \multicolumn{1}{l}{ Table 3: Hemorrhagic complications } & & & \\
\hline Complication & Patients & Total & Percentage \\
\hline Intraprocedural rupture & 12 & 339 & $4 \%$ \\
Postprocedural rerupture (within 1 month) & 4 & 339 & $1 \%$ \\
$\quad \begin{array}{l}\text { Intraparenchymal hemorrhage (not ventricular } \\
\quad \text { drain - related) }\end{array}$ & 1 & 339 & $<1 \%$ \\
$\begin{array}{l}\text { Extra-axial hemorrhage (not ventricular } \\
\quad \text { drain-related) }\end{array}$ & 1 & 339 & $<1 \%$ \\
$\begin{array}{l}\text { Ventricular drain-related hemorrhage } \\
\quad \text { (patients who had dual antiplatelet }\end{array}$ & 9 & $\geq 90$ & $\leq 10 \%$ \\
$\quad$ therapy) & & & \\
Total hemorrhagic complications & 27 & 339 & $8 \%$ \\
\hline
\end{tabular}

of $9(3 \%)$ of 339 patients. The total number of EVDs placed in these patients was not clear because 10 (63\%) of 17 articles, representing $228(76 \%)$ of 339 patients, did not report the total number of EVDs placed..$^{9-13,17,19,21-23}$ However, at least 90 ventricular drains in 9 case series were explicitly reported in patients who also received antiplatelet therapy. ${ }^{6,10,11,17,18,24-27}$ Thus, by using explicit data from these series, the calculated clinically significant ventricular drain - related bleeding complication rate was $9(10 \%)$ of 90 reported ventricular drain placements. Because we do not know the actual number of EVDs placed, the true bleeding complication rate was somewhere between $9 / 338(3 \%)$ and 9/90 (10\%), assuming all EVD-related bleeding complications were reported. Five $(56 \%)$ of 9 EVD-related hemorrhages were from a single case series. $^{25}$

Twelve (4\%) of 339 patients had intraprocedural rupture of aneurysms. ${ }^{6,20,22}$ More than half of these intraprocedural ruptures were reported in a single case series of 50 patients. ${ }^{20}$ Four (1\%) of 339 patients had confirmed or suspected rerupture of their aneurysm within 1 month after their procedure. ${ }^{6,20,25}$ One rupture occurred 14 days postprocedure, ${ }^{6}$ and 1 suspected rerupture occurred in a patient who rapidly decompensated and died. ${ }^{25}$ One patient had intraparenchymal hemorrhage that was not EVD-related. ${ }^{11}$ One patient had an extra-axial hemorrhage that was not EVD-related. ${ }^{23}$ In total, $\leq 18$ (5\%) of 339 patients had recognized or suspected bleeding complications not related to ventricular drains. Overall, $\leq 27(8 \%)$ of 339 patients had hemorrhagic complications during or after endovascular stent placement.

Data on thromboembolic complications specific to ruptured aneurysm cases were available in 14 (82\%) of 17 reports, comprising 288 patients (Table 4). 6,9,10,12,17-23,25-27 In-stent

\begin{tabular}{|c|c|c|c|}
\hline Complication & Patients & $\begin{array}{c}\% \text { for } \\
\text { Patients } \\
\text { with Data }\end{array}$ & $\begin{array}{c}\text { Proven } \% \text { for } \\
\text { Total } 339 \\
\text { Patients }\end{array}$ \\
\hline In-stent thrombosis & 1 & $0.3 \%(288)$ & $0.3 \%$ \\
\hline Clinically evident strokes & 15 & $5.2 \%(288)$ & $4.4 \%$ \\
\hline Total & 16 & $5.6 \%(288)$ & $4.7 \%$ \\
\hline
\end{tabular}

Table 5: Clinical presentation and outcomes

\begin{tabular}{|c|c|c|c|}
\hline \multirow[b]{2}{*}{ Clinical Outcomes } & \multicolumn{2}{|c|}{ Clinical Presentation } & \multirow[b]{2}{*}{ Total } \\
\hline & $\begin{array}{c}\text { Favorable } \\
\text { (HH I-III) }\end{array}$ & $\begin{array}{c}\text { Poor } \\
(\text { HH IV-V) }\end{array}$ & \\
\hline Favorable outcome (mRS I-II, GOS 1-2) & $171(81 \%)$ & $24(29 \%)$ & $195(67 \%)$ \\
\hline Poor outcome (mRS III-V, GOS 3-4) & $13(6 \%)$ & $28(34 \%)$ & $41(14 \%)$ \\
\hline Death (mRS VI, GOS 5) & $27(13 \%)$ & $30(37 \%)$ & $57(19 \%)$ \\
\hline Total & $211(72 \%)$ & $82(28 \%)$ & 293 \\
\hline
\end{tabular}

thrombosis occurred in 1 of 339 patients, which resulted in death. ${ }^{6}$ Fifteen $(5 \%)$ of 288 patients had clinically significant ischemic events. One of these infarcts resulted in death and occurred after aspirin was stopped 6 hours postprocedure due to femoral access site bleeding. In total, 16 (6\%) of 288 patients had thromboembolic complications thought to be related to stent placement.

Overall, including both hemorrhagic and thromboembolic complications, a total of $\leq 43$ (13\%) of 339 patients had clinically evident intracranial complications thought to be related to therapy. No patients had both intracranial hemorrhagic and thromboembolic complications. Surgical crossover was required after endovascular treatment in $6(2 \%)$ of 339 patients. Two of these 6 patients also had hemorrhagic complications listed above. Therefore, 292 (86\%) of 339 patients were treated successfully without complications or need for surgical crossover.

Clinical outcomes were available for 293 (86\%) of 339 patients (Table 5). ${ }^{6,9,10,12,17-22,24-27}$ One hundred six (36\%) of 293 patients had extended clinical follow-up averaging 9.5 months. The timing of clinical outcome data for the remaining 187 (64\%) patients was at discharge or unclear, so it was assumed to be at the time of discharge. Of these patients, 211 (72\%) had favorable initial presentations ( $\mathrm{HH}$ grade I-III) and $82(28 \%)$ had poor initial presentations ( $\mathrm{HH}$ grade IV-V). Of patients who had favorable initial presentation, $171(81 \%)$ had favorable clinical outcomes, $13(6 \%)$ had poor clinical outcomes, and $27(13 \%)$ died. Of patients who had poor initial presentation, $24(29 \%)$ had favorable clinical outcomes, 28 (34\%) had poor clinical outcomes, and 30 (37\%) died. Overall, 195 (67\%) had favorable clinical outcomes, 41 (14\%) had poor clinical outcomes, and 57 (19\%) died.

\section{Discussion}

In this literature review, we identified a substantial cohort of patients with ruptured aneurysms in whom stent-assisted coiling or stand-alone stent placement was performed acutely, usually with dual antiplatelet therapy during and in the months following intervention. Overall, we found a higher rate of intracranial hemorrhagic complications compared with thromboembolic complications. The overall procedurerelated complication rate including both hemorrhagic and 
thromboembolic complications was $\leq 13 \%$, which is higher than reported complication rates in stent-assisted coiling of unruptured aneurysms, which are in the $6 \%-7 \%$ range. ${ }^{28,29}$ Of particular concern is the risk for hemorrhage in patients who require EVD placement or conversion of EVD to a permanent shunt, given the need for ongoing antiplatelet therapy. In this review, however, hemorrhagic complications related to placement of EVDs were uncommon with the exception of the case series of a single center. However, reporting of EVD placement was inconsistent across studies, with no data on the presence or absence of EVD placement in $72 \%$ of patients. Additionally, the overall rate of hemorrhage in patients having ventricular drains in this series is similar ( $\leq 10.0 \%$ compared with $7.1 \%$ ) to a reported series of patients who had not received dual antiplatelet therapy. ${ }^{30}$ It is unknown whether the temporal relationship between the placement of ventricular drains and endovascular therapy has significant effect on the risk of hemorrhage. Another concern in patients on dual antiplatelet therapy arises if there is a need to convert an EVD to a permanent shunt. In our practice, when the need of converting an EVD to a shunt arises in a patient with aneurysmal SAH on dual antiplatelet therapy, we have usually placed the ventricular catheter for the shunt by using the same tract and burr hole as those for the EVD. This practice has proved safe and effective. ${ }^{31}$

Clinical outcomes in this literature review appear relatively poor compared with previous case series and trials of coil embolization without stent assistance. In a recently presented meta-analysis of 2 randomized clinical trials of aneurysm coiling (HydroCoil Endovascular Aneurysm Occlusion and Packing Study [HELPS] and CCT), good outcome at discharge for patients with good-grade ruptured aneurysms (World Federation of Neurological Societies [WFNS] 1-3) was 94\% (P.M. White, unpublished data, February 2010). Eighty-eight percent of the good-grade ruptured aneurysms in the International Subarachnoid Aneurysm Trial, an older randomized clinical trial of aneurysm coiling, had good clinical outcomes at discharge. ${ }^{1,2}$ Our systematic review demonstrates $81 \%$ good clinical outcome for good-grade aneurysms. This apparent difference in the current series compared with those prior reports is a cause for concern, especially when considering that randomized controlled trials report better outcomes compared with the relatively less rigorous self-reported data of this systematic review, which typically has an inherently positive outcomes bias. However, the subgroup analyzed in our review almost certainly constitutes a group of patients at higher risk because aneurysms treated with stents tend to have more complex morphology (eg, neck width, aneurysm shape, or location). Unfortunately, there are no data available for a comparison of similar patient populations.

Prolonged aspirin and dual antiplatelet therapy have been shown to increase the risk of both intra- and extracranial hemorrhage. Large meta-analyses of multiple trials of aspirin and dual platelet therapy in patients with cardiac disease have demonstrated that aspirin alone increases bleeding risk for both intra- and extracranial hemorrhage, with serious bleeding rates of $1.5 \%-2.5 \%$ for all aspirin doses ${ }^{32}$; and dual antiplatelet therapy increases the risk of all types of bleeding $40 \%-$ $50 \%$ further. ${ }^{33}$ This potential additional complication related to stent use is not captured for most of the patients in this report due to short-term clinical follow-up. In addition, there was some variation in technique, including types of stents used, technique for stent placement, anticoagulation regimens, and possibly differences in patient selection for stentassisted coiling versus traditional coiling or primary surgical intervention.

This study, even though it represents a compilation of all available stent-assisted coiling case series, has several limitations. First, the data are not from a single population but are a compilation of separate patient series published by different authors who reported independently assessed data, which has a high likelihood of publication bias, ${ }^{34-36}$ in different formats, and who focused their reports on different specific variables that are included in this article. Furthermore, this series includes only published cases. There may be a publication bias that excludes either patients who had uneventful outcomes or those who had failure or poor outcomes. Additionally, several of the reports did not differentiate bleeding complications within the elective nonruptured aneurysms from the acutely ruptured cases. Complications occurring $>30$ days postprocedure were not included in this report because most authors reported the results of the initial hospitalization. Therefore, complications from dual antiplatelet therapy after 30 days or after discharge from the hospital may be missing or underreported in these data.

\section{Conclusions}

Further investigation is certainly warranted before final conclusions can be made. Current published data suggest that adverse outcomes are more common in stent-assisted coil embolization in acutely ruptured aneurysms compared with similar grade acutely ruptured aneurysms treated by coil embolization alone. However, it remains unknown if this difference is due to technical differences or inherent differences in risk between 2 different patient populations.

Disclosures: Harry J. Cloft. Research Support (including provision of equipment or materials): Cordis; Mindframe. Details: Receive research support from Cordis Endovascular as enrolling center for SAPPHIRE carotid stent trial. Research support to read images for PRIISM study sponsored by Mindframe. Consultant. Medtronic. Details: Serve on Data Safety and Monitoring Board for KAVIAR trial sponsored by Medtronic. Giuseppe Lanzino. Research Support (including provision of equipment or materials): ev3; Synthes. Details: Unrestricted educational grants. Consultant. Actelion Inc, Edge Therapeutics. David J. Fiorella. Research Support (including provision of equipment or materials): National Institutes of Health. Details: SAMMPRIS grant. Consultant: Micrus Endovascular, Microvention. Details: $<\$ 10,000$. Ownership Interest. Revasc Inc (acquired by Micrus Endovascular). Details: $>\$ 10,000$, stroke revascularization device IP. Philip M. White. Research Support (including provision of equipment or materials). chief investigator of HELPS trial funded (but not sponsored by) by MicroVention Terumo Inc. Consultant. Consultancy work for Micrus and MicroVention for teaching and educational purposes. David F. Kallmes. Research Support (including provision of equipment or materials). NFocus, Sequent, MicroVention, ev3.

\section{References}

1. Molyneux A, Kerr R, Stratton I, et al. International Subarachnoid Aneurysm Trial (ISAT) of neurosurgical clipping versus endovascular coiling in 2143 patients with ruptured intracranial aneurysms: a randomised trial. Lancet 2002;360:1267-74

2. Molyneux AJ, Kerr RS, Yu LM, et al. International Subarachnoid Aneurysm Trial (ISAT) of neurosurgical clipping versus endovascular coiling in 2143 patients with ruptured intracranial aneurysms: a randomised comparison of effects on survival, dependency, seizures, rebleeding, subgroups, and aneurysm occlusion. Lancet 2005;366:809-17

3. Guglielmi G, Vinuela F, Sepetka I, et al. Electrothrombosis of saccular aneurysms via endovascular approach. Part 1. Electrochemical basis, technique, and experimental results. J Neurosurg 1991;75:1-7

4. Guglielmi G, Vinuela F, Dion J, et al. Electrothrombosis of saccular aneurysms 
via endovascular approach. Part 2. Preliminary clinical experience. J Neurosurg 1991;75:8-14

5. Higashida RT, Smith W, Gress D, et al. Intravascular stent and endovascular coil placement for a ruptured fusiform aneurysm of the basilar artery: case report and review of the literature. J Neurosurg 1997;87:944-49

6. Tahtinen OI, Vanninen RL, Manninen HI, et al. Wide-necked intracranial aneurysms: treatment with stent-assisted coil embolization during acute ( $<72$ hours) subarachnoid hemorrhage-experience in 61 consecutive patients. Radiology 2009;253:199-208

7. Ries T, Buhk JH, Kucinski T, et al. Intravenous administration of acetylsalicylic acid during endovascular treatment of cerebral aneurysms reduces the rate of thromboembolic events. Stroke 2006;37:1816-21

8. Lopes D, Sani S. Histological postmortem study of an internal carotid artery aneurysm treated with the Neuroform stent. Neurosurgery 2005;56:E416, discussion E416

9. dos Santos Souza MP, Agid R, Willinsky RA et al. Microstent-assisted coiling for wide-necked intracranial aneurysms. Can J Neurol Sci 2005;32:71-81

10. Katsaridis V, Papagiannaki C, Violaris C. Embolization of acutely ruptured and unruptured wide-necked cerebral aneurysms using the Neuroform 2 stent without pretreatment with antiplatelets: a single center experience. AJNR Am J Neuroradiol 2006;27:1123-28

11. Mocco J, Snyder KV, Albuquerque FC, et al. Treatment of intracranial aneurysms with the Enterprise stent: a multicenter registry. J Neurosurg 2009;110:35-39

12. Biondi A, Janardhan V, Katz JM, et al. Neuroform stent-assisted coil embolization of wide-neck intracranial aneurysms: strategies in stent deployment and midterm follow-up. Neurosurgery 2007;61:460-68, discussion 468-69

13. Lylyk P, Ferrario A, Pasbon B, et al. Buenos Aires experience with the Neuroform self-expanding stent for the treatment of intracranial aneurysms. $\mathrm{J} \mathrm{Neu}$ rosurg 2005; 102:235-41

14. Akpek S, Arat A, Morsi H, et al. Self-expandable stent-assisted coiling of widenecked intracranial aneurysms: a single-center experience. AJNR Am J Neuroradiol 2005;26:1223-31

15. Sani S, Jobe KW, Lopes DK. Treatment of wide-necked cerebral aneurysms with the Neuroform 2 Treo stent: a prospective 6-month study. Neurosurg Focus 2005;18:E4

16. Alfke K, Straube T, Dorner L, et al. Treatment of intracranial broad-neck aneurysms with a new self-expanding stent and coil embolization. AJNR Am J Neuroradiol 2004;25:584-91

17. Benitez RP, Silva MT, Klem J, et al. Endovascular occlusion of wide-necked aneurysms with a new intracranial microstent (Neuroform) and detachable coils. Neurosurgery 2004;54:1359-67, discussion 1368

18. Jankowitz B, Thomas AJ, Vora N, et al. Risk of hemorrhage in combined Neuroform stenting and coil embolization of acutely ruptured intracranial aneurysms. Interv Neuroradiol 2008;14:385-96. Epub 2009 Jan 5

19. Kim BM, Chung EC, Park SI, et al. Treatment of blood blister-like aneurysm of the internal carotid artery with stent-assisted coil embolization followed by stent-within-a-stent technique: case report. J Neurosurg 2007;107:1211-13

20. Kim SR, Vora N, Jovin T, et al. Anatomic results and complications of stentassisted coil embolization of intracranial aneurysms. Interv Neuroradio 2008;14:267-84

21. Klisch J, Eger C, Sychra V, et al. Stent-assisted coil embolization of posterior circulation aneurysms using Solitaire AB: preliminary experience. Neurosurgery 2009;65:258-66, discussion 266

22. Lee BH, Kim BM, Park MS, et al. Reconstructive endovascular treatment of ruptured blood blister-like aneurysms of the internal carotid artery. J Neurosurg 2009;110:431-36

23. Lee YJ, Kim DJ, Suh SH, et al. Stent-assisted coil embolization of intracranial wide-necked aneurysms. Neuroradiology 2005;47:680-89

24. Mordasini P, Walser A, Gralla J, et al. Stent placement in the endovascular treatment of intracranial aneurysms. Swiss Med Wkly 2008;138:646-54

25. Tumialan LM, Zhang YJ, Cawley CM, et al. Intracranial hemorrhage associated with stent-assisted coil embolization of cerebral aneurysms: a cautionary report. J Neurosurg 2008;108:1122-29

26. Yahia AM, Gordon V, Whapham J, et al. Complications of Neuroform stent in endovascular treatment of intracranial aneurysms. Neurocrit Care 2008;8: 19-30

27. Taylor RA, Callison RC, Martin CO, et al. Acutely ruptured intracranial saccular aneurysms treated with stent assisted coiling: complications and outcomes in 42 consecutive patients. J Neurointervent Surg 2010;2:23-30

28. Liang G, Li Z, Wei X, et al. Neuroform stent-assisted coiling of intracranial aneurysms: a 5 year single-center experience and follow-up. Neurol Res 2010;32:721-27

29. Piotin M, Blanc R, Spelle L, et al. Stent-assisted coiling of intracranial aneurysms: clinical and angiographic results in $\mathbf{2 1 6}$ consecutive aneurysms. Stroke 2010;41:110-15

30. Saladino A, White JB, Wijdicks EF, et al. Malplacement of ventricular catheters by neurosurgeons: a single institution experience. Neurocrit Care 2009;10: 155-56

31. Rammos S, Klopfenstein J, Augspurger L, et al. Conversion of external ventricular drains to ventriculoperitoneal shunts after aneurysmal subarachnoid hemorrhage: effects of site and protein/red blood cell counts on shunt infection and malfunction. J Neurosurg 2008;109:1001-04

32. Serebruany VL, Steinhubl SR, Berger PB, et al. Analysis of risk of bleeding complications after different doses of aspirin in 192,036 patients enrolled in 31 randomized controlled trials. Am J Cardiol 2005;95:1218-22

33. Serebruany V. Dose of aspirin and bleeding risks. Am J Cardiol 2006;98:275

34. Ergina PL, Cook JA, Blazeby JM, et al. Challenges in evaluating surgical innovation. Lancet 2009;374:1097-104

35. Rothwell P, Warlow C. Is self-audit reliable? Lancet 1995;346:1623

36. Rothwell PM. Interpretation of variations in outcome in audit of clinical interventions. Lancet 2000;355:4-5 